\title{
Impact Load and Mechanical Respond of Tibiofemoral Joint
}

\author{
A.A. Oshkour1, N.A. Abu Osman1, M.M. Davoodi1, M. Bayat1, Y.H. Yau2, and W.A.B. Wan Abas1 \\ 1 Department of Biomedical Engineering, Faculty of Engineering, University of Malaya, Malaysia \\ 2 Department of Mechanical Engineering, Faculty of Engineering, University of Malaya, Malaysia \\ INTRODUCTION
}

The human knee joint is the most important and biggest

joint in the body [1]. It has a complex structure and constructed

from different articulations and components, which

namely are femoral cartilage, tibia cartilages, ligaments and

menisci [2]. Knee tolerates large forces and moments during

different daily activity $[3,4]$. Hence, knee injury is a common

disease. Better understanding on knee joint biomechanics

can help to prevent injury to the knee. To date variety of

parameters analyzed via experimental measurements or

finite element studies [5]. Finite element (FE) methods as a

powerful tool in engineering have been employed by many

researchers to understand of knee biomechanics.

Yildirim et al. used FE analysis to determine and show the contact location in the knee during high flexion [4].

Finite element methods applied to analysis menisci and meniscectomy effect, and ligaments biomechanics, in knee

joint behaviour [2,6,7]. 3-dimentional (3D) FE was performed

to analysis contact pressure and compressive stress

in healthy human knee in flexion and gait $[1,8]$.

The knee joint impact is an action that we faced with it regularly during daily jumping and running or exercise.

Even many studies have been done on knee joint, still there are much unknown parameter should be considered for better underrating of knee joint especially during knee impact.

Therefore for the current study of impacting knee with rigid plate is considered to understand of the knee respond to impact load.

MATERIAL AND METHODS 
Two models are considered for current study as shown in

Fig. 1. In the model a, a circular plate hit the femur at the end $(\mathrm{P}-\mathrm{F})$ with initial velocity. Meanwhile, in model b, circular plate has impact with tibia (P-T). To perform the current study two main steps has been done (i) creating 3D model of TF and (ii) FEA.

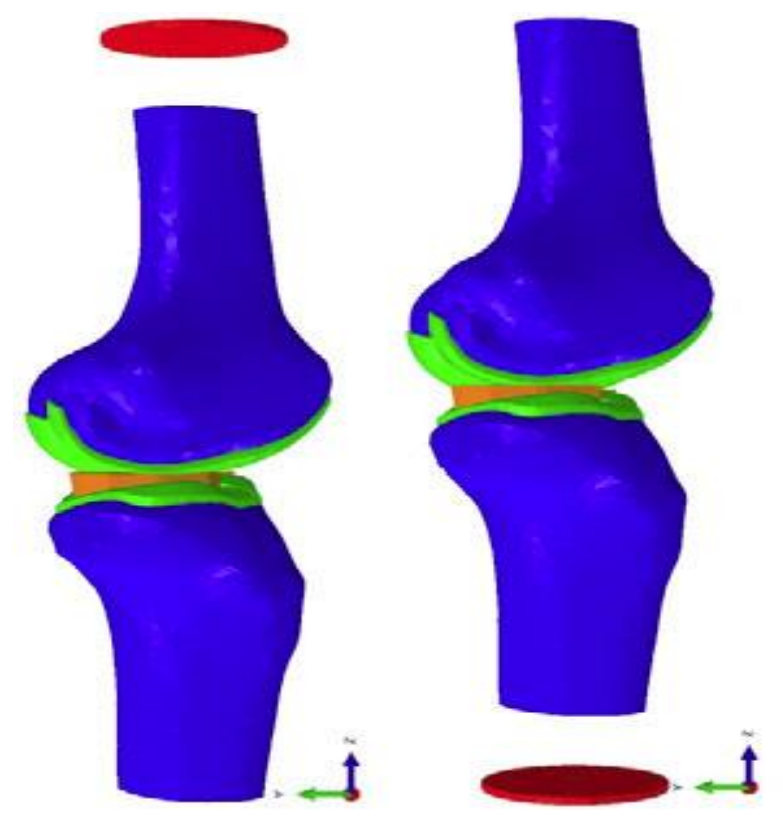

Fig. 1 Two tibiofemoral joint model

\section{A. Creating Tibiofemoral Joint}

To create 3D model Computed tomography (CT) were

used. The CT scan has performed on a 24-year-old healthy female with weight of $50 \mathrm{~kg}$ and height of $162 \mathrm{~cm} .988$ images were captured using a multidetector Siemens machine with $512 * 512$ pixels and a spatial resolution of $0.549 \mathrm{~mm}$.

CT images were converted using Digital Imaging and Communication (DICOM) formats and were then imported to the Mimics software. Soft and hard tissues were identified using tissue specific threshold values of 148-1872 and 125-700, respectively and the tibia, femur, cartilages, and menisci were represented in the knee model (maximum and minimum value of threshold corresponds to the range of 
grey values to highlight pixels). After creating the knee components in Mimics, the 3D model was imported as into ABAQUS finite element software.

B. Defining Material Properties

The properties were given to TF components based on literature review [1]. Bones (tibia and femur) were defined cortical bone, elastic and linear material with Young modulus of $11 \mathrm{GPa}$ and Poisson's ratio of 0.3 . Femoral and tibial cartilages were considered linear and elastic material with a Young's modulus of $5 \mathrm{MPa}$ and a Poisson's ratio of 0.46 . Menisci were also considered elastic with a Young's modulus of $59 \mathrm{MPa}$ and a Poisson's ratio of 0.49 .

\section{Loading and Boundary Conditions}

A rigid plate with initial velocity of $60 \mathrm{~mm} / \mathrm{s}$ was hit the end of the femur and tibia in the P-F and P-T respectively. The distance of $20 \mathrm{~mm}$ was considered between rigid plate and its target (femur or tibia). Femur allowed moving vertically in PF and it has been fixed at the end in P-T. At the same time, tibia was fixed at the P-F and the allowed moving vertically in P-T. Cartilages were perfectly attached to the corresponding bones, and the motion of the menisci was restricted to the lateral and medial direction. The frictionless contact properties are considered between all model components.

Full text is available at :

http://download.springer.com/static/pdf/846/chp\%253A10.1007\%252F978-3-642-21729-

6 44.pdf?auth66=1393724079_d520841ffce131ca148737180b697a60\&ext $=. p d f$

http://link.springer.com/chapter/10.1007/978-3-642-21729-6 44 